\title{
Harmonic Superspaces and Superconformal Fields
}

\author{
Paul Heslop and Paul Howe. \\ The Maths_Dept, King's College, The Strand, London, WC2R 2LS, England \\ E-mail:pheslop@mth.kcl.ac.uk
}

ABstRACT: Representations of four-dimensional superconformal groups on harmonic superfields are discussed. It is argued that any representation can be given as a superfield on any (non twistorial) superflag manifold. Representations on analytic superspaces do not require constraints. We discuss short representations and how to obtain them as explicit products of fundamental fields. We also discuss superfields that transform under supergroups.

\section{Introduction}

The unitary irreducible representations of superconformal groups have assumed more significance recently in the light of the Maldacena conjecture [1]1] relating string theory or M-theory on $A d S \times S$ to superconformal field theories on the boundary. A particularly important class of operators that can arise consists of those operators which correspond to short representations of the superconformal group since these are expected to be protected from quantum corrections and thus not acquire anomalous dimensions [2]. Long representations are also of interest and are supposed to correspond to string states [3]

There are different methods of constructing

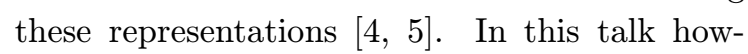
ever we discuss a way of constructing representations explicitly as superfields using the method of parabolic induction, focusing on four dimensional superconformal groups, $S U(2,2 \mid N){ }^{1}$ This

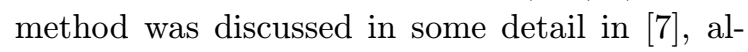
though a direct comparison with the more al-

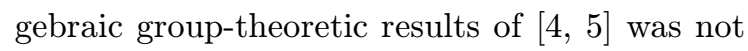
made at the time. We complexify spacetime, and complexify the superconformal group to $S L(4 \mid N)$ so that all the spaces of interest become coset spaces of this group. We claim that any representation may be given as a holomorphic field on any superflag space (except for supertwistor spaces).

\footnotetext{
${ }^{1}$ Superconformal fields in harmonic superspaces have also been discussed recently in [6].
}

On some spaces (e.g. super Minkowski space and chiral spaces) the fields will require extra constraints, whereas on others (in particular analytic spaces) they will require no constraints, and this makes tensoring representations together to produce other representations straightforward.

In section 2 we briefly recall parabolic induction and illustrate this in the bosonic context with the group $S L(N)$. In section 3 we consider the full superconformal group $S L(4 \mid N)$ and look at short representations. Finally in section 4 we consider representations that transform under supergroups, and give some specific examples.

This talk is based on [iإئin and work in progress.

\section{The Bosonic case}

\subsection{Coset Spaces}

If $G$ is a Lie group and $P$ a subgroup, a coset space $M$ is the space of (right) cosets: $M=P \backslash G$, and we obtain the fibre bundle: $G \rightarrow P \backslash G$, with fibres $\mathrm{P}$.

We can define a representation of $G$ on the space of equivariant maps $F: G \rightarrow V$, where $V$ is the representation space of $P$, i.e. maps such that

$$
F(h u)=R(h) F(u)
$$

where $u \in G$ and $R$ is the representation of $P$ on $V$ (in practice these are fields with indices). The induced representation itself is given by $F \mapsto$ 
$g \cdot F, g \in G$ where

$$
(g \cdot F)(u)=F(u g) .
$$

All the subgroups $P$ we are interested in will be parabolic subgroups (defined below), and the maps $F$ will be holomorphic maps. In this case the spaces are known as flag spaces.

Let $\mathrm{G}$ be a complex, simple Lie group and let $\mathfrak{g}$ be its Lie algebra. For the case of $\mathfrak{s l}(N)$ we define the Borel subalgebra to be the algebra of all lower triangular matrices (with non-zero entries on the diagonal allowed), and a parabolic subalgebra $\mathfrak{p}$ is one which is block lower triangular:

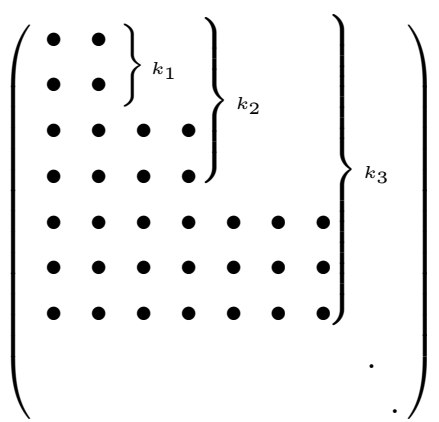

The corresponding Levi subalgebra is the block diagonal subalgebra, i.e. $\mathfrak{s}\left(\mathfrak{g l} l\left(k_{1}\right) \oplus \mathfrak{g l}\left(k_{2}-k_{1}\right) \oplus\right.$ ...). A parabolic $\mathfrak{p}$ can also be represented by placing a cross on each of the nodes $k_{1}, k_{2}, \ldots, k_{l}$ of the Dynkin diagram for $\mathfrak{s l}(N)$ (see $[\bar{i}$ i $)$ ).

For example, complexified Minkowski space can be viewed as an open subset of the coset space $P \backslash S L(4), S L(4)$ being the complexified conformal group and $P$ the parabolic subgroup of matrices of the following shape:

$$
\left(\begin{array}{llll}
\bullet & \bullet & & \\
\bullet & \bullet & & \\
\bullet & \bullet & \bullet & \bullet \\
\bullet & \bullet & \bullet & \bullet
\end{array}\right)
$$

where the bullets denote elements which do not have to be zero. The blank region can be thought of as corresponding to spacetime. Indeed, we can choose a coset representative of the form

$$
M \ni x \mapsto s(x)=\left(\begin{array}{cc}
1_{2} & x \\
0_{2} & 1_{2}
\end{array}\right)
$$

where each entry is a two-by-two matrix. From this one can easily work out the transformation of $x$ under the conformal group. The Levi subalgebra is $\mathfrak{s}(\mathfrak{g l} l(2) \oplus \mathfrak{g l}(2))$ and the Dynkin diagram is $\bullet \leftrightarrow \bullet$.

\subsection{Representations of $S L(N)$}

Highest weight representations of $S L(N)$ can be specified by giving $N-1$ integral Dynkin labels, $a_{i} \geq 0, i=1 \ldots N-1$ which are placed above the nodes of the Dynkin diagram for $S L(N)$. Highest weight representations of parabolic subgroups are actually representations of the Levi subgroup as the other bits act trivially. These can also be specified by giving $N-1$ Dynkin labels, placed above the corresponding Dynkin diagram with crosses through it (in this case the labels above nodes with crosses through them can be negative, and correspond to $\mathbb{C}$ charges, the remaining labels give the representations of the $\mathfrak{s l}\left(k_{i}\right)$ of the Levi algebra.) The Borel-Weil theorem tells us that if we pick a representation of $P$ with positive Dynkin labels, then the induced representation of $G$ on holomorphic fields of $P \backslash G$ described above is isomorphic to the representation of $G$ with the same Dynkin labels (see [ig $\left[\begin{array}{l}0 \\ 0\end{array}\right]$. Diagrammatically we have

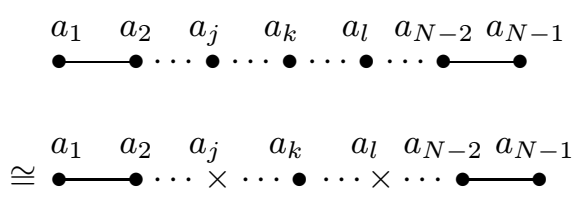

where crosses can be placed on any of the nodes of the right hand side.

The simplest example of this formula gives representations of $S L(2)$ as fields on $H \backslash S L(2)=$ $\mathbb{C P}^{1}$ where $H$ is the set of $2 \times 2$ lower triangular matrices with unit determinant. Diagrammatically we have

$$
\begin{aligned}
& p \quad 0 \quad p \\
& -\quad \times
\end{aligned}
$$

The right hand side of this equation represents the space of holomorphic tensor fields of charge $p$ on $\mathbb{C} P^{1}$. This is a $p+1$-dimensional space which is identified with the space of $p$ th rank symmetric tensors under $S L(2)$ (the left-hand side of the equation.)

\section{Superspaces}

We wish to extend the above formalism to the 
case of the superconformal group $S L(4 \mid N) .^{2}$ Here we can have different, inequivalent Borel subgroups (see $10 \overline{1}$ ). However, if we make a change of basis of $\mathbb{C}^{\overline{4} \mid N}$ on which the $S L(4 \mid N)$ matrix acts, we can change an element in the Lie algebra $\mathfrak{s l}(4 \mid N)$ as follows:

$$
g=\left(\begin{array}{l|l}
4 & \\
&
\end{array}\right) \rightarrow\left(\begin{array}{l|l|l}
2 & & \\
\hline & N & \\
\hline & & 2
\end{array}\right) .
$$

In this basis the particular choice of Borel subalgebra consistent with super Minkowski space consists of the lower triangular matrices, and parabolic subalgebras consist of block lower triangular matrices, as in the bosonic case.

Super Dynkin diagrams can also be defined for $S L(4 \mid N)$ as follows

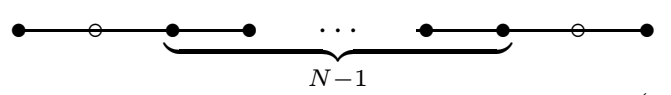

In this diagram the $N-1$ central black nodes represent the $\mathfrak{s l}(N)$ subalgebra. The two black nodes on the ends represent the space-time $\mathfrak{s l}(2)$ representations. The two white nodes are odd nodes representing odd roots in the Lie algebra. This diagram is not a unique diagram for $\mathfrak{s l}(4 \mid N)$, but is the one which corresponds to the choice of Borel subalgebra above, and is thus the one which is consistent with super Minkowski space. Different Borel subalgebras will lead to different Dynkin diagrams. Crosses can be put anywhere on this diagram to represent parabolic spaces as in the bosonic case.

For example, complexified super Minkowski space has the form $P \backslash S L(4 \mid N)$ where $P$ consists of matrices of the form

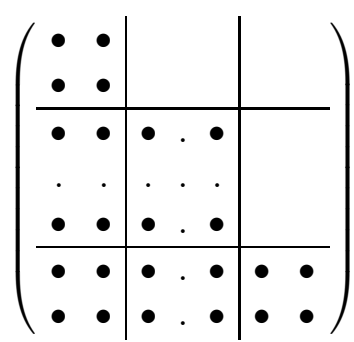

\footnotetext{
${ }^{2}$ We are eventually going to be interested in unitary representations of the real superconformal group $S U(2,2 \mid N)$. However, homogeneous space techniques are more easily applied in the complex setting. One can return to real spacetime by taking $x$ real and $\varphi=\bar{\theta}$.
}

and has corresponding Dynkin diagram

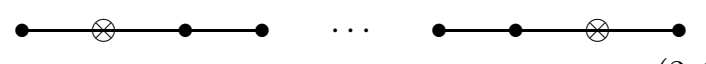

The standard coset representative is

$$
M \ni z \mapsto s(z)=\left(\begin{array}{c|c|c}
1_{2} & \theta & x \\
\hline 0 & 1_{N} & \varphi \\
\hline 0 & 0 & 1_{2}
\end{array}\right)
$$

where $\varphi$ denotes the $N$ dotted two-component spinorial coordinates which become the complex conjugates of the $\theta$ 's in the real case.

We shall be interested in other superspaces which extend Minkowski space by an internal flag space. Such superspaces are called harmonic superspaces and were first introduced by GIKOS [1]1]. Complexified $(N, p, q)$ harmonic superspace has the following Dynkin diagram

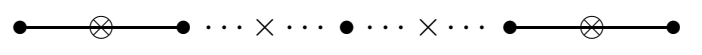

where the middle crosses are on the $p$ th and $(N-$ $q)$ th central nodes. Locally, this space has the form of complex super Minkowski space times an internal flag space. The related $(N, p, q)$ analytic superspace has the same body but fewer odd coordinates. It has the following Dynkin diagram

This space has only $(N-p) \theta$ 's and $(N-q)$ $\varphi$ 's. Generalised $(N, p, q)$ spaces can be defined, which have the same number of $\theta$ 's and $\varphi$ 's as $(N, p, q)$ space, but have a different internal space. These are given by the same Dynkin diagram as above, but with any number of extra crosses inserted between the two already there.

\subsection{Superconformal Representations}

Representations of the superconformal group $S L(4 \mid N)$ can be specified by the following quantum numbers: Lorentz spin, $j_{1}, j_{2}$, dilation weight, $L$, R-charge $R$, and the Dynkin labels of the internal group, $a_{1} \ldots a_{N-1}$. The unitary irreducible highest weight representations fall in three

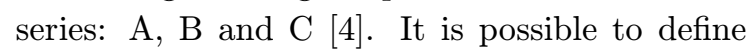
super-Dynkin labels for the group $S L(4 \mid N)$ as 
follows:

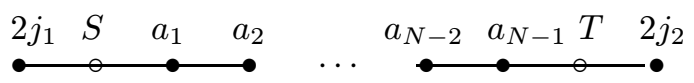

where

$$
\begin{aligned}
& S=\frac{1}{2}(L-R)+j_{1}+\frac{m}{N}-m_{1} \\
& T=\frac{1}{2}(L+R)+j_{2}-\frac{m}{N}
\end{aligned}
$$

and where $m_{1}=\sum_{k=1}^{N-1} a_{k}, m=\sum_{k=1}^{N-1} k a_{k}$. Then the three series correspond to the following conditions on the labels:

$$
\begin{array}{lll}
\text { A) } & S \geq 2 j_{1}+1 & T \geq 2 j_{2}+1 \\
\text { B) } & S \geq 2 j_{1}+1 & T=j_{2}=0 \\
\text { or } & S=j_{1}=0 & T \geq 2 j_{2}+1 \\
\text { C) } & S=j_{1}=0 & T=j_{2}=0
\end{array}
$$

We are now in a position to apply the formalism of section 2 to the super case.

\subsection{Short Representations}

Short representations are characterised by being short multiplets and thus having shorter range of spins than unconstrained superfields on Minkowski superspace. Such representations act naturally on superfields defined on analytic superspaces since these have fewer odd coordinates than Minkowski superspace.

The superfields on $(N, p, q)$ space should transform under irreducible representations of Levi subalgebras of the form $\mathfrak{l}=\mathfrak{s}(\mathfrak{g l}(2 \mid p) \oplus \mathfrak{g l}(2 \mid q) \oplus$ $\mathfrak{g l}(r)), r=N-(p+q)$. However, in order to ensure that the representations are indeed short these superfields must not carry any spacetime indices. They must therefore transform trivially under any supergroup factors of the Levi subgroup. In the generic case this means that they transform only under $\mathfrak{s l}(r) \oplus \mathbb{C}^{2}$.

In order to keep matters as simple as possible, we shall concentrate for the time being on $(N, p, q)$ superspaces. The representations to be studied can then be represented by modified Dynkin diagrams of the following type:

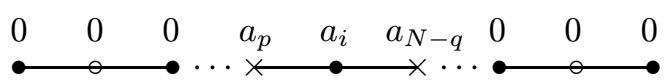

For the reasons discussed above the first $(p-1)$ and the last $(q-1)$ Dynkin labels must vanish, leaving $(r-1)$ labels to specify the representation of the central $\mathfrak{s l}(r)$ and two further labels which specify the charges.

\subsection{Massless Multiplets}

Some simple examples of superconformal representations are given by on-shell massless multiplets, with maximal helicity s, where $\left[\frac{N}{2}\right] \leq 2 s<$ $N\left(\left[\frac{N}{2}\right]\right.$ denotes the nearest integer greater than or equal to $\frac{N}{2}$.) These have the following superDynkin labels:

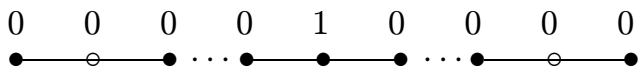

where $a_{p}=1$ and all other Dynkin labels are 0 (here $p=2 s$ ). These are described in (real) super Minkowski space, $M$, by superfields $W$ which have $p$ totally antisymmetric internal indices and which satisfy $\left[12^{1} 1,1,13^{1}\right]$

$$
\begin{aligned}
\bar{D}_{\dot{\alpha}}^{i} W_{j_{1} \ldots j_{p}} & =\frac{p(-1)^{p-1}}{N-p+1} \delta_{\left[j_{1}\right.}^{i} \bar{D}_{\dot{\alpha}}^{k} W_{\left.j_{2} \ldots j_{p}\right] k} \\
D_{\alpha i} W_{j_{1} \ldots j_{p}} & =D_{\alpha[i} W_{\left.j_{1} \ldots j_{p}\right]}
\end{aligned}
$$

For each such superfield there is a conjugate superfield $\tilde{W}_{i_{1} \ldots i_{N-p}}$, and these obey similar constraints. When $s=\frac{1}{4} N$, the multiplet is selfconjugate.

We can extend this to $p=N$, since for such a superfield the constraints (13.13i) imply that it is anti-chiral. Its conjugate has no indices and is chiral. Such a chiral field describes an onshell massless super multiplet (with maximum spin $N / 2$ ) if it satisfies the additional constraint

$$
D_{\alpha i} D_{j}^{\alpha} W=0
$$

The most natural spaces to put these representations on is $(N, p, N-p)$ analytic superspace, giving the Dynkin diagram

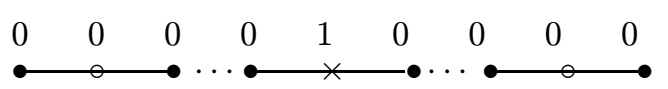

On these spaces they don't satisfy any constraints (an example of this will be given in the next section.) 
There are many other ways of representing such multiplets which are "less efficient" in that the superspaces have more odd coordinates. Following the discussion at the end of the previous section we can simply place crosses where we like on the above Dynkin diagram with the restriction, for the moment, that the cross furthest to the left must be to the left of the node with the 1 above it, and the cross furthest to the right must be to the right of this node, to avoid super indices. As we are talking about analytic superspaces, we do not want any crosses on the end nodes, (these would correspond to super twistor spaces) . For example, any such field (excluding $p=0, N)$ can be realised on $(N, 1,1)$ space:

To illustrate this procedure we consider the $N=4$ Maxwell super multiplet which is represented on $N=4$ super Minkowski space by the self-conjugate Sohnius superfield $W_{i j}$ [1] 191$]$. We can put this on $(4,2,2)$ analytic space [1 15 n]

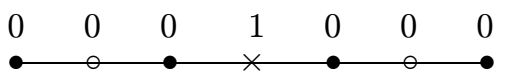

where it becomes a field with no indices, and a charge. In the Yang-Mills theory, we can multiply these together and get fields which correspond to the Kaluza-Klein states on $A d S^{5}$ in the $A d S / C F T$ correspondence. We could also, however put it on $(4,1,1)$ analytic superspace [1] $\left.{ }^{1}\right]$

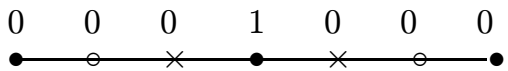

on which it has an $S L(2)$ index: $W_{1 r}, r \in\{2,3\}$. We can obtain all series $\mathrm{C}$ representations with $R=0$ by simply multiplying copies of this field, and taking irreducible representations of $S L(2)$. Finally we could put it on $(4,1,0)$ analytic superspace

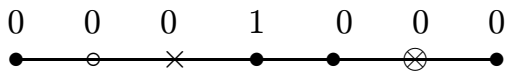

where it becomes a field with an $S L(3)$ index: $W_{1 r}, r \in\{2,3,4\}$. In this case, the field only obeys all the constraints of the Sohnius field due to self-conjugacy. We can obtain all series $\mathrm{C}$ and B representations with $R=0$ by multiplying copies of this field together and taking irreducible representations under $S L(3)$. Doing this using the Yang-Mills field and taking multiple traces corresponds to BPS states in the $A d S / C F T$ cor-

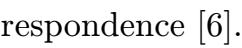

The above superfields have been defined on $(4,1,1)$ and $(4,1,0)$ superspaces which have the smallest possible internal flags. It is possible to relax this, and use "generalised" $(N, p, q)$ spaces. For example we could use the maximal flag space determined by the Borel subalgebra. In the $(4,1,1)$ case for instance, this is the space

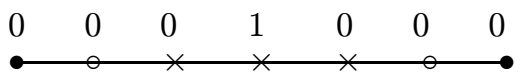

We would then split the indices as $I=1,2,3,4$; this enables us to define the field $W_{12}$. A disadvantage of this is that multiplying such fields together to get different representations is now no longer such a simple procedure.

\section{Super Indices}

In the previous section we considered various short representations on harmonic superspaces, and we insisted that they did not have any super indices so we could explicitly see that the representations were short. In this section we consider fields that do have super indices. For simplicity we will restrict ourselves to $N=2$ analytic superspace. This has the Dynkin diagram

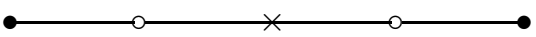

and corresponding parabolic subgroup of the form

$$
\left(\begin{array}{ll}
a_{B}^{A} & 0 \\
c_{A^{\prime} B} & d_{A^{\prime}} B^{\prime}
\end{array}\right)
$$

where each entry is a $(2 \mid 1) \times(2 \mid 1)$ matrix. The Levi subalgebra (under which our fields transform) is $\mathfrak{s l}(2 \mid 1) \oplus \mathfrak{s l}(2 \mid 1) \oplus \mathbb{C}$ (corresponding to the block diagonals), where the first $\mathfrak{s l}(2 \mid 1)$ subalgebra is carried by un-primed indices, and the second by primed indices. We may choose a local coset representative $s(X)$, as follows:

$$
s(X)=\left(\begin{array}{cc}
1 & X \\
0 & 1
\end{array}\right) \in S L(4 \mid 2)
$$


where each element is a $(2 \mid 1) \times(2 \mid 1)$ matrix, and

$$
X=\left(\begin{array}{cc}
\lambda^{\alpha} & x^{\alpha \dot{\alpha}} \\
y & \pi^{\dot{\alpha}}
\end{array}\right) \text {. }
$$

The important point here is that it takes two coordinate patches to cover analytic superspace. If we denote these two sets by $U$ and $U^{\prime}$ and put primes on the coordinates for $U^{\prime}$ then in the overlap, the two sets are related as follows:

$$
\begin{aligned}
x^{\prime} & =x-\frac{\lambda \pi}{y}, \\
\lambda^{\prime} & =-\frac{1}{y} \lambda, \\
\pi^{\prime} & =\frac{1}{y} \pi, \\
y^{\prime} & =\frac{1}{y} .
\end{aligned}
$$

Requiring our fields to be holomorphic on both patches puts restrictions on the fields, which are equivalent to the constraints on Minkowski space.

We illustrate this first, briefly, with the example of the hypermultiplet [i] i i . This is the representation of $S L(4 \mid 2)$ with dilation weight $L=1, R=0, j_{1}=j_{2}=0$ but has a non-zero Dynkin label $a_{1}=1$. It therefore has the following Dynkin diagram on analytic superspace:

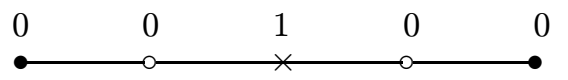

We read from this diagram that the field is invariant under both $\mathfrak{s l}(2 \mid 1)$ subalgebras (i.e. our field has no super indices), but it does have a $\mathbb{C}$ charge. Thus our field is specified by two local holomorphic functions $W(x, \lambda, \pi, y)$ and $W^{\prime}\left(x^{\prime}, \lambda^{\prime}\right.$, on $U$ and $U^{\prime}$ respectively, such that in the overlap $U \cap U^{\prime}$

$$
W(x, \lambda, \pi, y)=y W^{\prime}\left(x^{\prime}, \lambda^{\prime}, \pi^{\prime}, y^{\prime}\right) .
$$

From this one can show that it has only a short expansion:

$$
\begin{aligned}
W(x, \lambda, \pi, y) & =\varphi_{1}(x)+y \varphi_{2}(x)+\lambda^{\alpha} \psi_{\alpha}(x) \\
& +\pi^{\dot{\alpha}} \chi_{\dot{\alpha}}(x)-\lambda^{\alpha} \pi^{\dot{\alpha}} \partial_{\alpha \dot{\alpha}} \varphi_{2}
\end{aligned}
$$

with all the components satisfying their equations of motion. This is the usual hypermultiplet with two complex scalar fields and two complex Weyl fermions, all of which are physical and onshell.

Consider next the $N=2$ on-shell Maxwell multiplet, which is usually a chiral field in super Minkowski space. This has dilation weight
1, R-charge - 1 and all other quantum numbers 0 . The Dynkin diagram for this field on analytic superspace is

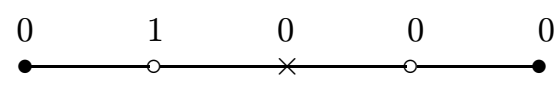

We can read off from the above Dynkin diagram exactly how the fields transform under the Levi subalgebra. In particular, it transforms non-trivially under the first $\mathfrak{s l}(2 \mid 1)$ superalgebra. In fact, we have a field with one down-stairs un-primed super index. Again we have two local holomorphic fields on $U$ and $U^{\prime}$ which we denote $W_{A}=$ $\left(W_{\alpha}, W\right)$ and $W_{A}^{\prime}=\left(W_{\alpha}^{\prime}, W^{\prime}\right)$, and one can show that these are related as follows in the intersection:

$$
\begin{aligned}
W_{\alpha} & =y W_{\alpha}^{\prime} \\
W & =W^{\prime}-\frac{1}{y} \lambda^{\alpha} W_{\alpha}
\end{aligned}
$$

giving us the following result:

$$
\begin{aligned}
W_{\alpha} & =\rho_{1 \alpha}+y \rho_{2 \alpha}+\lambda^{\beta} F_{\alpha \beta}-\pi^{\dot{\alpha}} \partial_{\alpha \dot{\alpha}} C \\
& -\lambda^{\beta} \pi^{\dot{\beta}} \partial_{\beta \dot{\beta}} \rho_{2 \alpha} \\
W & =C-\lambda^{\alpha} \rho_{2 \alpha}
\end{aligned}
$$

again with all the components satisfying their equations of motion. These components are all on-shell and we have reproduced the $N=2$ Maxwell multiplet which is given on Minkowski space by a chiral field satisfying the second order con$\pi^{\prime}, y^{\prime}$ traint $\left(13 . \overline{1} \overline{4^{\prime}}\right)$.

As a final example, consider the $N=2$ superconformal stress-energy multiplet. On super Minkowski space it is a scalar superfield $T$ satisfying

$$
D_{\alpha i} D_{j}^{\alpha} T=0 .
$$

It has Dilation weight 2, and all other quantum numbers are 0 . On analytic superspace it has the Dynkin diagram

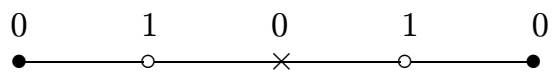

and from this we see that it is given by the superfield $T_{A^{\prime} A}$. It has been explicitly checked that this does indeed give the correct on-shell components. This representation can be obtained explicitly in two different ways on analytic superspace: firstly by multiplying a Maxwell field and 
its conjugate together

$$
T_{A^{\prime} A}=W_{A^{\prime}} W_{A}
$$

and secondly by multiplying two hypermultiplet fields together with a derivative:

$$
T_{A^{\prime} A}=W_{1} \partial_{A^{\prime} A} W_{2}-W_{2} \partial_{A^{\prime} A} W_{1} .
$$

\section{Conclusion}

In this talk we have shown how to obtain representations of the superconformal group on certain coset spaces of this group. We claim that any representation may be given as a tensor field on almost any superflag manifold, if we allow the fields to transform under supergroups. On some spaces the fields may require constraints, whereas on others, in particular analytic superspaces, no constraints are required. This facilitates the tensoring together of different representations. The super Dynkin diagrams provide a simple way of giving all the information required for putting representations on coset spaces.

Superfield representations of the superconformal group are important in the $A d S / C F T$ correspondence. For example, we believe it is possible to obtain all $N=4$ superconformal representations explicitly by multiplying copies of the Maxwell superfield and applying derivatives on $(4,2,2)$ analytic superspace. The formalism should be useful when considering correlation functions in super Yang Mills theories [1 $\left.\overline{7}_{1}\right]$. There also appears to be a similarity with the oscillator construction of superconformal representations [5]

Acknowledgements. P. Heslop thanks the TMR network and the organisers of the conference for support.

\section{References}

[1] J. Maldacena, The large $N$ limit of superconformal field theories and supergravity, Adv. Theor. Math. Phys. 2 (1998) 231-252, hep-th/9711200

[2] S. Ferrara and A. Zapparoni, Superconformal fields, multiplet shortening and the $\mathrm{AdS}_{5} / \mathrm{SCFT}_{4}$ correspondence hep-th/9908163

[3] L Andrianopoli and S Ferrara On short and long $S U(2,2 \mid 4)$ multiplets in the AdS/CFT correspondence, Lett. Math. Phys. 48 (1999) 145, hep-th/9812067.
[4] M.Flato and C. Fronsdal, Lett. Math. Phys. 8 (1984) 159; V.K. Dobrev and V.B. Petkova, Phys. Lett. B162 (1985) 127, Fortschr. Phys. 35 (1987) 537; B. Binegar, Phys. Rev. D34 (1986) 525; B. Morel, A. Sciarrino and P. Sorba, Phys. Lett B166 (1986) 69, erratum B167 (1986) 486.

[5] M Gunaydin, D Minic, M Zagermann 4D Doubleton Conformal Theories, CPT and IIB String on $A d S_{5} \times S^{5}$, Nucl.Phys. B534 (1998) 96-120

[6] S. Ferrara and E. Sokatchev, Short representations of $S U(2,2 \mid N)$ and harmonic superspace analyticity. hep-th/9912168; S. Ferrara and E. Sokatchev, Superconformal Interpretation of BPS States in AdS Geometries hep-th/0005151

[7] P.S. Howe and G.G. Hartwell, A superspace survey, Class. Quant. Grav. 12 (1995) 1823-1880.

[8] P. Heslop and P.S.Howe On Harmonic Superspaces and Superconformal Fields in Four Dimensions, hep-th/0005135

[9] R Baston and M Eastwood, The Penrose Transform, Oxford University Press 1989.

[10] J.F.Cornwell Group Theory in Physics vol.III, Academic Press 1989

[11] A Galperin A, E Ivanov, S Kalitzin, V Ogievetsky and E Sokatchev, Unconstrained $N=2$ matter, Yang-Mills and supergravity theories in harmonic superspace, Class. Quantum Grav. 1 (1984) 469

[12] W. Siegel, Nucl. Phys. B177 (1981) 325.

[13] P.S. Howe, K.S. Stelle and P.K. Townsend, Nucl. Phys. B181 (1981) 445; Nucl. Phys. B182 (1981) 332.

[14] M.F. Sohnius, Nucl. Phys. 136 (1978) 461.

[15] G.G. Hartwell and P.S. Howe $(N, p, q)$ harmonic superspace, Int J. Mod. Phys 10, (1995) 39013919.

[16] I.A. Bandos Solution of linear equations in harmonic variables, Theor.Math.Phys. 76 no. 2 (1988) 169-183.

[17] P.S. Howe, E. Sokatchev and P.C. West, Threepoint functions in $N=4$ Yang-Mills, Phys. Lett. B444 (1998) 341-351, hep-th/9808162; B. Eden, P.S. Howe, C. Schubert, E. Sokatchev and P.C. West, Extremal correlators in four-dimensional SCFT, Phys. Lett. B472 (2000) 323-331, hepth/991015 